\title{
The Kidney Score Platform for Patient and Clinician Awareness, Communication, and Management of Kidney Disease: Protocol for a Mixed Methods Study
}

Delphine S Tuot ${ }^{1}$, MD; Susan T Crowley ${ }^{2,3}$, MD, MBA; Lois A Katz ${ }^{4,5}$, MD; Joseph Leung ${ }^{4,5}$, MD; Delly K Alcantara-Cadillo ${ }^{2,3}$, MD; Christopher Ruser ${ }^{2,3}$, MD; Elizabeth Talbot-Montgomery ${ }^{6}$, BSc; Joseph A Vassalotti ${ }^{7}$, MD

${ }^{1}$ University of California, San Francisco, San Francisco, CA, United States

${ }^{2}$ Veterans Affairs Connecticut Healthcare System, West Haven, CT, United States

${ }^{3}$ Yale University School of Medicine, New Haven, CT, United States

${ }^{4}$ Veterans Affairs New York Harbor Healthcare System, New York, NY, United States

${ }^{5}$ New York University Grossman School of Medicine, New York, NY, United States

${ }^{6}$ National Kidney Foundation, New York, NY, United States

${ }^{7}$ Icahn School of Medicine at Mount Sinai, New York, NY, United States

\section{Corresponding Author:}

Delphine S Tuot, MD

University of California, San Francisco

Zuckerberg San Francisco General Hospital

1001 Potrero Ave

San Francisco, CA, 94110

United States

Phone: 16282063784

Email: Delphine.tuot@ucsf.edu

\section{Abstract}

Background: Patient awareness, clinician detection, and management of chronic kidney disease remain suboptimal, despite clinical practice guidelines and diverse education programs.

Objective: This protocol describes a study to develop and investigate the impact of the National Kidney Foundation Kidney Score Platform on chronic kidney disease awareness, communication, and management, by leveraging the Behavior Change Wheel, an implementation science framework that helps identify behavioral intervention targets and functions that address barriers to behavior change.

Methods: We interviewed 20 patients with chronic kidney disease and 11 clinicians to identify patient and clinician behaviors suitable for intervention and barriers to behavior change (eg, limited awareness of chronic kidney disease clinical practice guidelines within primary care settings, limited data analytics to highlight chronic kidney disease care gaps, asymptomatic nature of chronic kidney disease in conjunction with patient reliance on primary care clinicians to determine risk and order kidney testing). Leveraging the Behavior Change Wheel, the Kidney Score Platform was developed with a patient-facing online Risk Calculator and a clinician-facing Clinical Practice Toolkit. The Risk Calculator utilizes risk predictive analytics to provide interactive health information tailored to an individual's chronic kidney disease risk and health status. The Clinical Practice Toolkit assists clinicians in discussing chronic kidney disease with individuals at risk for and with kidney disease and in managing their patient population with chronic kidney disease. The Kidney Score Platform will be tested in 2 Veterans Affairs primary health care settings using a pre-post study design. Outcomes will include changes in patient self-efficacy for chronic kidney disease management (primary outcome), quality of communication with clinicians about chronic kidney disease, and practitioners' knowledge of chronic kidney disease guidelines. Process outcomes will identify usability and adoption of different elements of the Kidney Score Platform using qualitative and quantitative methods.

Results: As of September 2020, usability studies are underway with veterans and clinicians to refine the patient-facing components of the Kidney Score Platform before study initiation. Results and subsequent changes to the Kidney Score Platform will be published at a later date. The study is expected to be completed by December 2021 . 
Conclusions: Results of this study will be used to inform integration of the Kidney Score Platform within primary care settings so that it can serve as a central component of the National Kidney Foundation public awareness campaign to educate, engage, and empower individuals at risk for and living with chronic kidney disease.

International Registered Report Identifier (IRRID): PRR1-10.2196/22024

(JMIR Res Protoc 2020;9(10):e22024) doi: 10.2196/22024

\section{KEYWORDS}

chronic kidney disease; CKD; awareness; implementation science; behavioral change wheel, RE-AIM

\section{Introduction}

Chronic kidney disease affects 37 million Americans [1]. Chronic kidney disease progression can ultimately lead to kidney failure, a life-threatening illness that, even with dialysis treatment, confers a death rate worse than most cancers and significantly reduces quality of life [2]. Additionally, chronic kidney disease is a disease multiplier that often occurs with diabetes or hypertension and increases the risk of emergency department visits, hospitalizations, and cardiovascular events [3]. Total Medicare spending for beneficiaries with chronic kidney disease and kidney failure was over $\$ 120$ billion in 2017 , of which over $\$ 35.9$ billion was spent to manage or treat kidney failure, a condition that can often be prevented with optimal chronic kidney disease management [2].

Chronic kidney disease is usually asymptomatic. Optimal management slows the progression of kidney disease and reduces cardiovascular events [4,5]. Individuals cannot readily know their disease status or risk without clinician recognition of risk, testing, detection, and communication [6]. Previous studies $[7,8]$ show that clinician detection and communication about kidney disease are suboptimal. Importantly, clinician diagnosis of chronic kidney disease is associated with increased delivery of evidence-based care, as well as increased patient awareness of their kidney disease [9-11]. As many as half of patients with advanced chronic kidney disease are unaware that they have chronic kidney disease, including those with laboratory manifestations of their kidney disease [12]. Patient awareness of chronic kidney disease, including the knowledge of having a kidney problem and the perceived risk of developing kidney disease, as well as the ability to affect their kidney health, is necessary for patients to participate in shared decision making about their kidney health and to apply management recommendations to improve outcomes [13]. Existing education programs, awareness campaigns, and clinical practice guidelines, including those from the National Kidney Foundation's (NKF) Kidney Disease Outcomes Quality Initiative and the Veterans Affairs and Department of Defense Clinical Practice Guidelines for the Management of Chronic Kidney Disease, have minimally improved chronic kidney disease awareness in the United States and veteran populations, respectively $[14,15]$.

Among the population served by Veterans Affairs (VA), 1 in 6 has chronic kidney disease. It is the fourth most diagnosed disease within the Department of Veteran Affairs, and over 13,000 veterans develop end-stage renal disease each year [16]. Diagnosis within the VA is low, with only $39 \%$ of veterans with chronic kidney disease stages 3-4 appropriately identified with International Classification of Diseases diagnostic codes in 2011 [7]. A roundtable discussion during the Kidney Innovation Summit hosted by the VA Center for Innovation and the American Society of Nephrology highlighted the need for a paradigm shift in education and awareness by addressing gaps in communication between "what is said, what is heard, and what the patient understands" and educating clinicians on how to meaningfully engage with patients at different stages of kidney health. This investigation is a significant incremental contribution to the Advancing American Kidney Health Initiative's aim 1 of 3 to reduce the number of Americans developing end-stage renal disease by $25 \%$ by 2030 [17].

Implementation science frameworks, which take into account multiple interacting domains and processes that factor into successful program implementation, can help assure that the aforementioned gaps in chronic kidney disease awareness and education are addressed with interventions that are feasible and generalizable in real-world setting [18]. Consistent with these principles, the National Kidney Foundation developed the Kidney Score Platform to increase individual awareness and perceived risk of chronic kidney disease, thereby enhancing discussions about kidney disease between patients and clinicians. With this paper, we describe how we leveraged an implementation science framework to develop the Kidney Score Platform and propose to implement and evaluate its impact on veteran understanding and engagement with chronic kidney disease care as well as clinician self-efficacy for identifying and discussing chronic kidney disease with their patients.

\section{Methods}

\section{Behavior Change Wheel}

We selected the Behavior Change Wheel [19] as a framework for the development of the Kidney Score Platform. This framework was developed from a synthesis of 19 other frameworks of behavior change and incorporates 3 layers to guide intervention development and deployment (Figure 1) [19]. The first layer uses the Capability, Opportunity, Motivation, Behavior (COM-B) model to identify barriers, enablers, and sources of behaviors that serve as potential intervention targets. The second layer of the wheel identifies 9 intervention functions that could be leveraged by the Kidney Score Platform to address the enablers and barriers to behavior change identified in the first layer. The third layer offers 7 policy options that can be deployed to support the testing and subsequent use of the Kidney Score Platform. 
Figure 1. The behavior change wheel from reference Michie and colleagues [19].

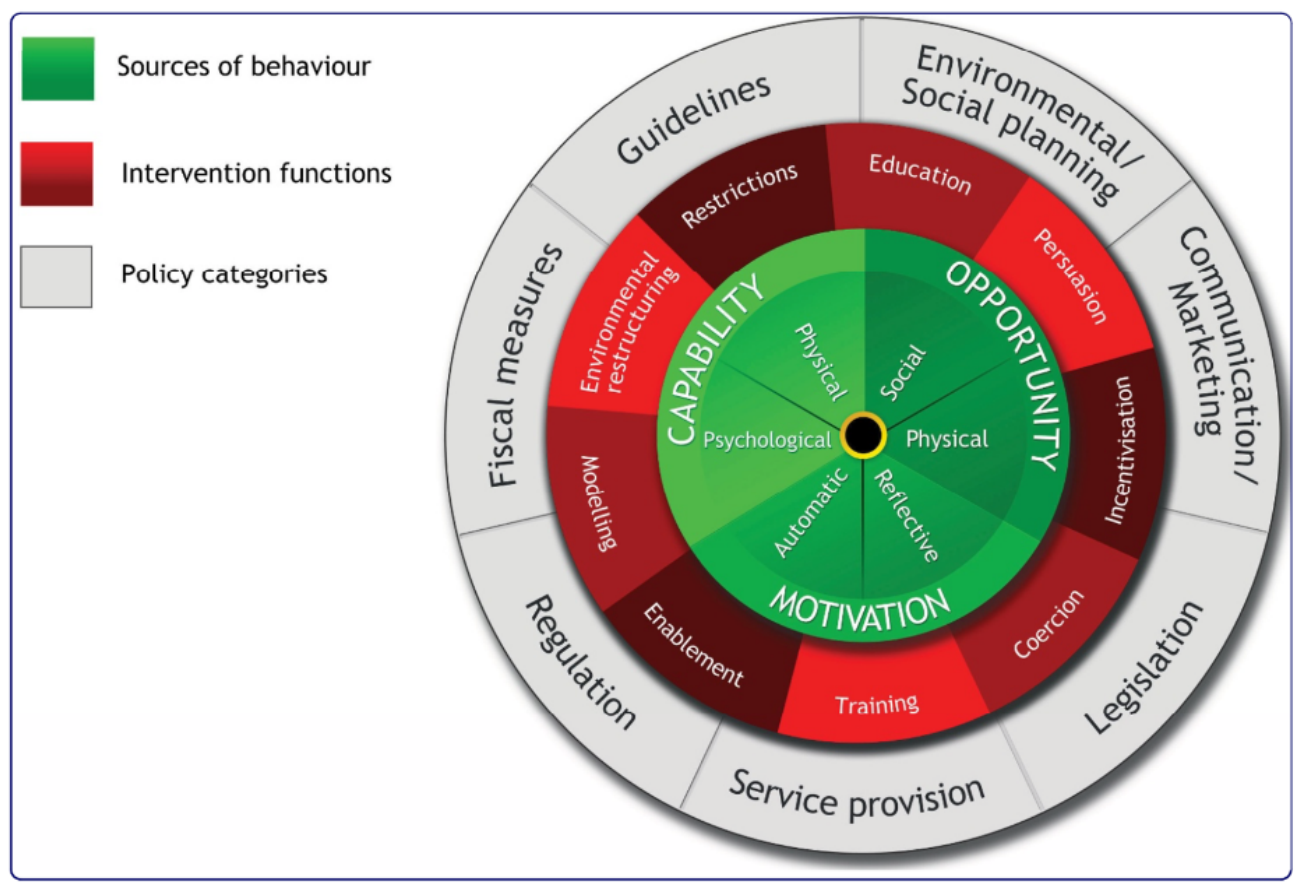

\section{The Kidney Score Platform}

From June 2016 to September 2016, the NKF conducted in-depth interviews with 20 nondialysis-requiring chronic kidney disease patients and 11 clinician experts in chronic kidney disease care including nephrologists and primary care practitioners (Multimedia Appendix 1). These interviews identified information that patients found most useful at various stages of their chronic kidney disease journey and provided insight into the type of information that clinicians conveyed to patients at those stages. Data from both clinician and patient audiences also identified barriers and enablers to behavior change that were categorized according to the COM-B model. Examples of patient barriers included the asymptomatic nature of disease, reliance on primary care clinicians to determine risk and order testing, and inadequate kidney-focused clinical workforce (ie, primary care clinicians, chronic kidney disease certified nutritionists, and nephrologists) (capability); underdiagnosis of chronic kidney disease in primary care and limited public awareness of chronic kidney disease (opportunity); and limited kidney literacy and low patient activation (motivation). Patient enablers included access to patient portals containing laboratory findings, availability of chronic kidney disease care planning, and care coordination (capability); availability of online chronic kidney disease patient education and access to chronic kidney disease care management tools (opportunity); and participation in chronic kidney disease peer mentoring programs and availability of self-management programs for chronic kidney disease (motivation).

Examples of clinician barriers were limited awareness of chronic kidney disease clinical practice guidelines, challenges in modifying organizational workflows for diabetes and hypertension, no financial incentives for chronic kidney disease recognition (capability); limited availability of chronic kidney disease training in medical school and absence of formal chronic kidney disease awareness campaigns (opportunity); low prioritization of chronic kidney disease by health care organizations and by health care payers; and limited data analytics to highlight chronic kidney disease care gaps (motivation). Clinician enablers included chronic kidney disease clinical decision support in the electronic health record, performance measures for chronic kidney disease testing, and risk adjustment strategies for chronic kidney disease diagnosis and severity (capability); systemic quality improvement focused on chronic kidney disease care processes and inclusion of chronic kidney disease interventions in risk factor education (ie, hypertension) (opportunity); and use of practice facilitation teams to improve chronic kidney disease care and use of registries to demonstrate opportunities in chronic kidney disease care (motivation).

\section{Kidney Score Platform Online Interface (for Patients)}

Based on the aforementioned behavior change targets, the NKF's multidisciplinary team of experts in web technology design, user interface development, information architecture, adult learning, and patient education partnered with experts in kidney care, patient education, and app design to develop the Kidney Score Platform online interface. Using the Behavior Change Wheel intervention functions education, persuasion, and enablement, the interface was designed to improve awareness and understanding about kidney disease among people at risk for and living with chronic kidney disease (Table 1). 
Table 1. Elements of the Kidney Score Platform informed by the 3 layers of the Behavior Change Wheel framework to directly address patient and clinician barriers and facilitators of behavior change for chronic kidney disease awareness, communication, and management.

\begin{tabular}{|c|c|c|c|c|c|}
\hline \multirow[t]{2}{*}{ Elements } & \multicolumn{2}{|l|}{ Behavior change } & \multicolumn{3}{|c|}{ Behaviour Change Wheel } \\
\hline & Barriers & Facilitators & Behavior sources & Intervention functions & Policy categories \\
\hline \multicolumn{6}{|l|}{ Patient-facing } \\
\hline \multicolumn{6}{|l|}{ Risk online interface } \\
\hline & Asymptomatic nature & $\begin{array}{l}\text { Patient portals with } \\
\text { laboratory data }\end{array}$ & Capability & Education & $\begin{array}{l}\text { Communication/mar- } \\
\text { keting }\end{array}$ \\
\hline & $\begin{array}{l}\text { Limited public aware- } \\
\text { ness }\end{array}$ & Online education & Opportunity & Education & $\begin{array}{l}\text { Communication/mar- } \\
\text { keting }\end{array}$ \\
\hline & Underdiagnosis & $\begin{array}{l}\text { Access to manage- } \\
\text { ment tools }\end{array}$ & Opportunity & Persuasion & Regulation \\
\hline & $\begin{array}{l}\text { Limited health litera- } \\
\text { cy; low patient activa- } \\
\text { tion }\end{array}$ & $\begin{array}{l}\text { Existing self-manage- } \\
\text { ment programs }\end{array}$ & Motivation & Enablement & Regulation \\
\hline \multicolumn{6}{|l|}{ Clinician-facing } \\
\hline Practice assessment & $\begin{array}{l}\text { Suboptimal awareness } \\
\text { of clinical practice } \\
\text { guidelines }\end{array}$ & $\begin{array}{l}\text { Quality improvement } \\
\text { focused on chronic } \\
\text { kidney disease }\end{array}$ & Opportunity & Persuasion & $\begin{array}{l}\text { Regulation; communi- } \\
\text { cation/ marketing }\end{array}$ \\
\hline CKDinform 2.0 & $\begin{array}{l}\text { Absence of formal } \\
\text { awareness campaigns }\end{array}$ & $\begin{array}{l}\text { Linkage of chronic } \\
\text { kidney disease with } \\
\text { risk factor education }\end{array}$ & Opportunity & Education & Guidelines \\
\hline AHRQ REALM-SF ${ }^{\mathrm{a}}$ & $\begin{array}{l}\text { Poor knowledge about } \\
\text { health literacy }\end{array}$ & $\begin{array}{l}\text { Validated tools to } \\
\text { quickly assess health } \\
\text { literacy }\end{array}$ & Motivation & Education & Regulation \\
\hline Teach-back video & $\begin{array}{l}\text { Limited training in } \\
\text { medical school }\end{array}$ & $\begin{array}{l}\text { Linkage of chronic } \\
\text { kidney disease with } \\
\text { risk factor education }\end{array}$ & Capability & Modeling & $\begin{array}{l}\text { Communication/mar- } \\
\text { keting; Regulation }\end{array}$ \\
\hline Change Package & $\begin{array}{l}\text { Challenges in modify- } \\
\text { ing workflows for dia- } \\
\text { betes and hyperten- } \\
\text { sion }\end{array}$ & $\begin{array}{l}\text { Clinical decision sup- } \\
\text { port in the electronic } \\
\text { health record }\end{array}$ & Capability & Enablement & Service provision \\
\hline
\end{tabular}

${ }^{a}$ AHRQ REALM-SF: Agency for Healthcare Research and Quality Rapid Estimate of Adult Literacy in Medicine—Short Form.

The 3 other policy categories described in the Behavior Change Wheel that will not be applied to the evaluate the Kidney Score Platform are fiscal, legislation, and social planning.

The free online interface utilizes a rule engine and risk predictive analytics to provide interactive health information tailored to the individual's chronic kidney disease risk and health status. After entering risk factor information as well as laboratory findings in the Kidney Score Platform's online interface, end users receive educational programming tailored to their current clinical status and risk for chronic kidney disease development or progression. Information is organized into context-specific easily digestible snippets, an approach aligned with the growing body of health education on how interactive content and tools such as apps, online health assessments, calculators, games, and quizzes can significantly affect comprehension, attitudes, self-efficacy, and health-related behavior change (Figure 2) [20-22]. The Kidney Score Platform Online interface blends the two of the most frequently accessed areas on the NKF website [23] (which receive over 19 million unique visitors per year) - the chronic kidney disease Risk Stratification Tool (Heat Map) and the patient-directed A to Z Guide-into a single tailored learning experience about kidney health. Topics span the spectrum of information from risk factors for chronic kidney disease to the benefit of medical nutrition therapy, fitness, weight control, and informed decision making for medical management of chronic kidney disease. 
Figure 2. Design of the Kidney Score Platform online interface, which utilizes a rule engine and risk predictive analytics to provide interactive health information tailored to the individual's chronic kidney disease risk and health status. CKD: chronic kidney disease; eGFR: estimated glomerular filtration rate; DM: diabetes; HTN: hypertension; uACR: urine albumin-creatinine ratio.

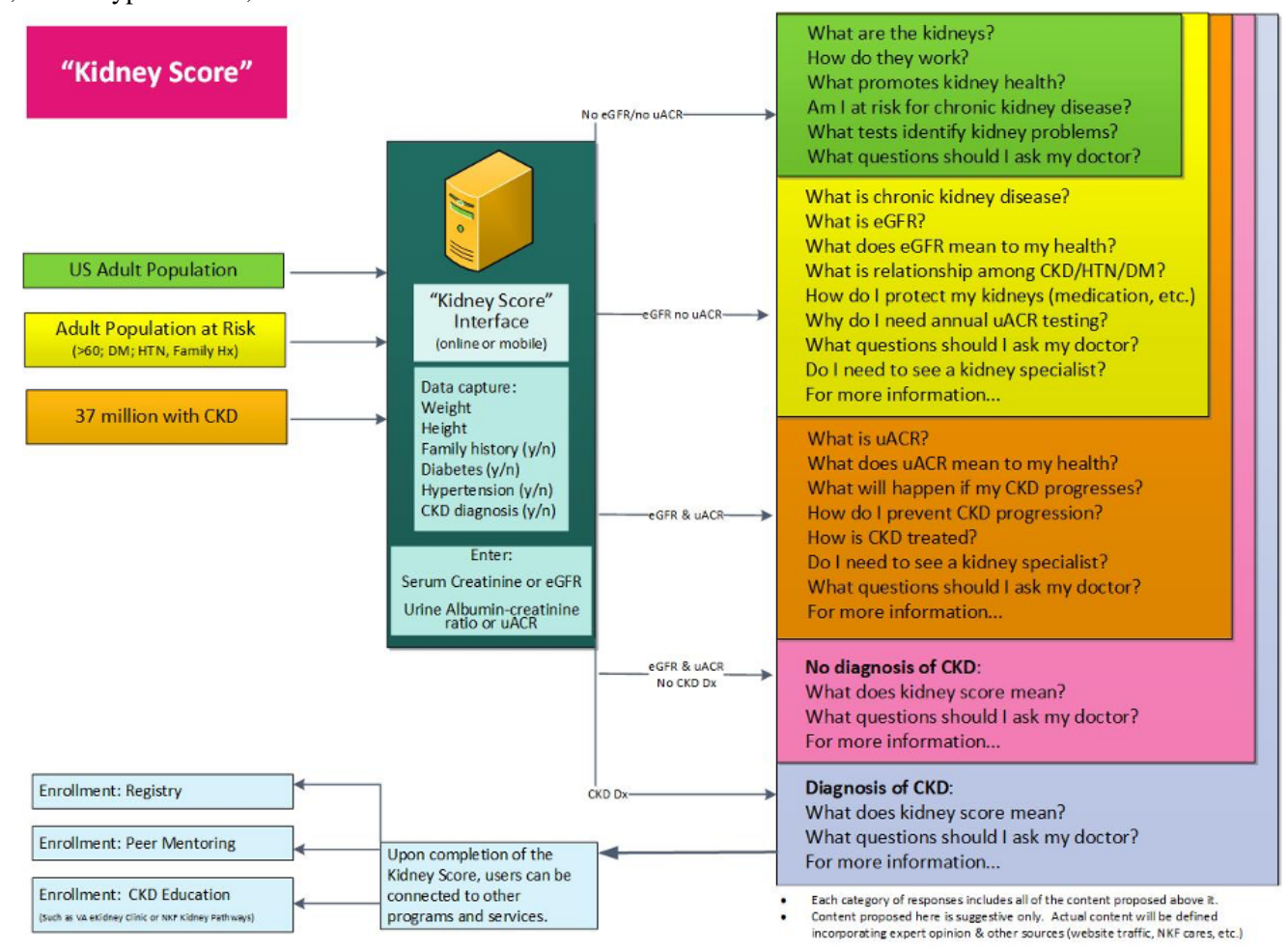

\section{Clinical Practice Toolkit (for Clinician Teams)}

Clinician-identified barriers and enablers for optimal discussions about chronic kidney disease informed the creation of the Kidney Score Platform's Clinical Practice Toolkit. Leveraging the Behavior Change Wheel intervention functions of education, persuasion, enablement, and modeling, the toolkit assists clinicians in discussing chronic kidney disease with individuals at risk of and those living with chronic kidney disease who may have different health literacy levels (Table 1). The toolkit includes elements that help identify patient populations at risk for and with chronic kidney disease, online continuing medical education about kidney disease detection and management, a tool that can be applied in clinical practice to assess patient health literacy for education tailoring, teach-back videos that model discussions about chronic kidney disease concepts, and quality improvement interventions that can be implemented at the clinic level to improve identification of patients at risk or living with chronic kidney disease.

Practice assessment is an algorithm that extracts chronic kidney disease-related data from the electronic medical record to assist clinical practices in identifying (1) patients at risk for chronic kidney disease who have not been tested for kidney disease; (2) patients with underlying undiagnosed chronic kidney disease based on laboratory testing; (3) patients with chronic kidney disease who could benefit from improved understanding of chronic kidney disease and the choices for medical management of chronic kidney disease.

CKDinform 2.0 is an online continuing education activity based on Kidney Disease Outcomes Quality Initiative clinical practice guidelines that review chronic kidney disease testing, detection, and care [24].
The Rapid Estimate of Adult Literacy in Medicine-Short Form (REALM-SF) is a validated 7-item word recognition test that provides clinicians with a quick assessment of a patient's health literacy and capacity to understand health information [25].

Teach-back videos provide examples for practitioners of how to utilize the Kidney Score Platform online tool to communicate with individuals at risk for or living with chronic kidney disease. These short videos, featuring an expert clinician investigator and an individual living with chronic kidney disease, provide several examples of the teach-back method being utilized to assess patient comprehension of chronic kidney disease-related concepts (ie, understanding the importance of kidney health, medical nutrition therapy for chronic kidney disease, and importance of avoiding certain over-the-counter medications) including instructions conveyed during the office visit about medication safety use.

Change Package is a free publicly available online compendium of tools or actionable process improvements that clinicians in primary care settings can implement to integrate Kidney Score Platform chronic kidney disease education and related interventions into their practice workflow [26]. By including process improvements that can be rapidly tested, the Change Package can help practices to deploy systems that efficiently and effectively support patients with chronic kidney disease. It was developed in collaboration with the Centers for Disease Control and Prevention Million Hearts initiative based on the foundation of their Hypertension Change Package [27].

\section{Study Design}

The policy strategies from the Behavior Change Wheel that enable and support the testing and use of the Kidney Score Platform include communication/marketing, regulation, 
guidelines, and service provision (Table 1). These are embedded in the pre-post study design that will test the impact of the Kidney Score Platform on patient self-efficacy for chronic kidney disease management within the primary health care settings of 2 VA medical centers: VA New York Harbor Healthcare System and VA Connecticut Healthcare System. This study has been approved by the Institutional Review Boards of the VA Connecticut Healthcare System (\#02290) and VA New York Harbor Healthcare System (\#01705) and any modifications to the study protocol will be communicated to them for review before implementation.

\section{Intervention}

Veterans will interact with the Kidney Score Platform online interface individually while awaiting their clinical encounter via tablets or laptops (communication/marketing). As depicted in Figure 3, veterans who have consented to participate in the study will complete an online preassessment survey before exposure to the Kidney Score Platform's Online Interface. Participants will then engage with the online interface for 5-20 minutes before their clinician visit. This interaction can occur at home for a telehealth visit or in the waiting room for an in-person visit. If requested, Kidney Score Platform will offer participants a copy of questions to ask their clinician about their kidney health, tailored to their chronic kidney disease risk, determined by the Kidney Score Platform's embedded rules engine. These questions aim to further reinforce important chronic kidney disease topics and foster meaningful patient-professional conversations (regulation). Within a week after the clinical encounter, veterans will be asked to complete a brief survey (electronically or by phone) to assess the impact of the Kidney Score Platform on their understanding of chronic kidney disease and the quality of their conversation with their practitioner about kidney health. The Kidney Score Platform is intended to serve as an adjunct to clinical care; there will be no restrictions on concomitant care interventions delivered during or after the clinical visit.

Prior to veteran recruitment, we will offer the accompanying clinical practice toolkit to primary care teams (service provision). Practitioners will be encouraged (but not mandated) to utilize the clinical practice tools, particularly completing CKDinform 2.0 and watching the chronic kidney disease teach-back video (communication/marketing). These tools will prepare practitioners for conversations with veterans as well as reinforce chronic kidney disease clinical practice guidelines on detection, patient education, and intervention (regulation, guidelines). Pre and postsurveys will be conducted to assess the impact of the clinical practice toolkit on practitioners' perception of chronic kidney disease, chronic kidney disease knowledge, and important topics for chronic kidney disease patient education.

Figure 3. Process flow for Kidney Score study. CKD: Chronic Kidney Disease; eGFR: estimated glomerular filtration rate; VHA: Veteran's Health Administration.

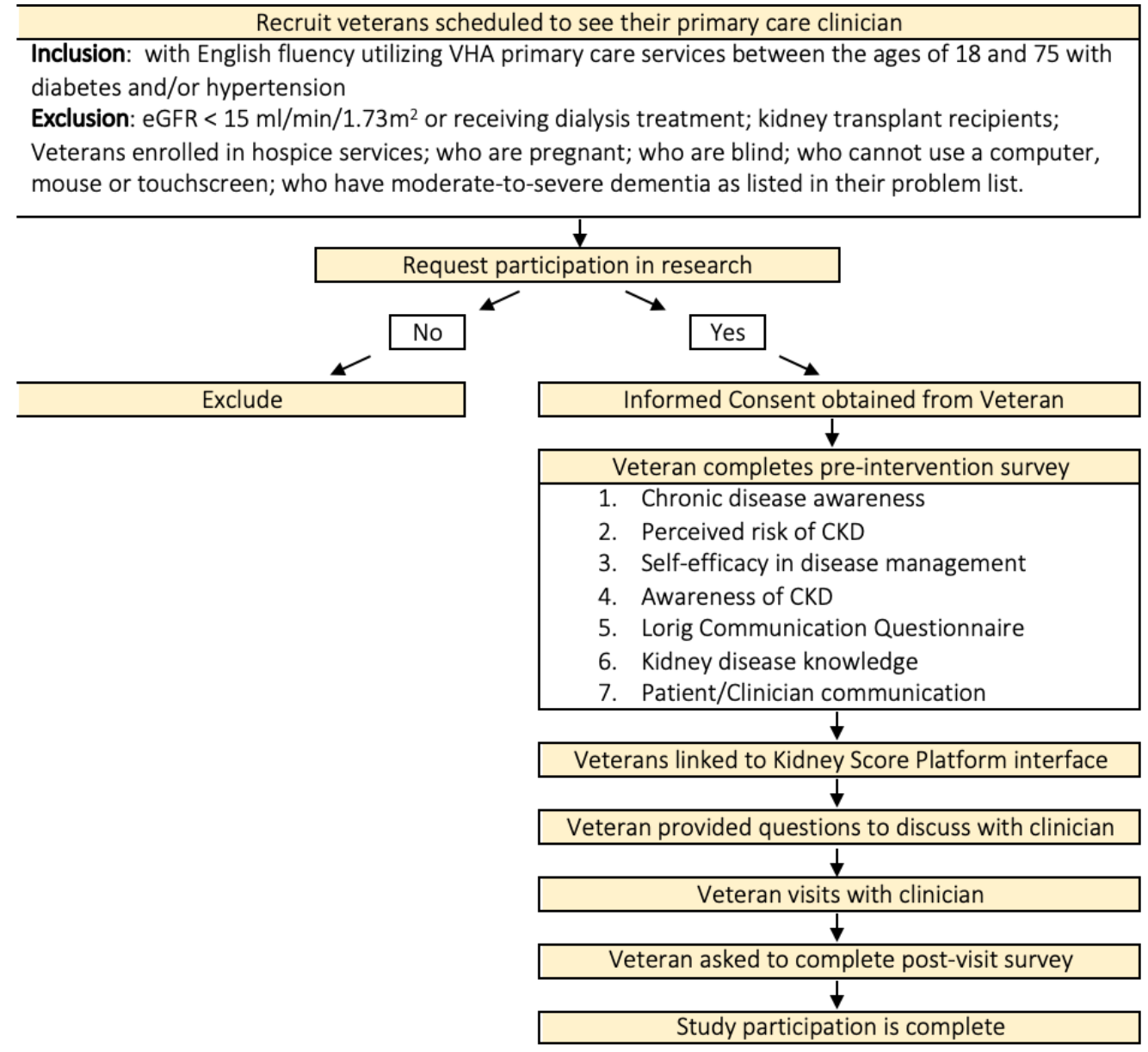




\section{Study Population Criteria}

Veterans with and at risk for chronic kidney disease and their primary care clinical teams represent the target population. English-speaking veterans utilizing VA primary care services between the ages of 18 and 75 with diabetes or hypertension as defined in the electronic health record will be eligible for this study. Exclusion criteria include an estimated glomerular filtration rate $<15 \mathrm{~mL} / \mathrm{min} / 1.73 \mathrm{~m}^{2}$ from recent laboratory tests in the medical record or those receiving dialysis treatment, as the Kidney Score Platform is not geared toward individuals with severe chronic kidney disease. Kidney transplant recipients will also be excluded, as their care is delivered predominantly in the subspecialty care setting rather than the primary care setting. We will also exclude veterans enrolled in hospice services, as chronic kidney disease awareness and self-management are less important for this population. Other exclusions include pregnancy, vision impairment, and severe dementia, identified by the problem list in the electronic health record or during the consent process. All primary care clinicians working as part of a Patient Aligned Care Team (PACT) in participating VA Medical Centers will be eligible for participation in the study.

\section{Recruitment}

Veterans work collaboratively with their PACT multidisciplinary team members to meet their health care needs. PACTs often include clinicians, nurses, medical assistants, social workers, and pharmacists, among other allied health professionals. The study team will work with each PACT team's care coordination staff to identify veterans who meet the inclusion criteria with appointments during the intervention's phase. Collaborating with primary care champions at each site, the project team will explore variations in recruitment process based on the site's resources, clinical flow, and use of telehealth for care delivery due to the COVID-19 pandemic.

Recruitment of clinicians from each PACT team will occur during a presentation of the project, during which the research team can answer questions. Clinical champions from each participating VA have been engaged in project co-design and will help ensure adequate and diverse clinician participation.

\section{Consent}

A member of the project team will describe the innovation and its goals and purpose to eligible veterans. Individuals that can correctly explain the project protocol and answer simple teach-back questions (in part to assess for dementia) will be asked to provide consent. Eligible providers will also be asked to provide consent to participate after they hear about the project and its goals.

\section{Data Collection and Outcomes}

\section{Sociodemographic Data}

Sociodemographic data (age, gender, race/ethnicity), co-morbid conditions (diabetes, hypertension, cardiovascular disease), and baseline laboratory data (serum creatinine, proteinuria, or albuminuria), will be collected from all consenting participants from the electronic health record, including participants who do not complete surveys for primary outcome collection (see below). All individually identifiable data will remain within the VA data system. Practitioner sociodemographic data (age, gender, race/ethnicity), role, panel size or practitioner caseload, and years of experience will be self-reported.

\section{Behavior and Attitude Outcomes}

The primary outcome is change in self-efficacy for chronic kidney disease management, measured by the Patient Activation Measure [28]. We will also examine changes in self-reported communication with practitioners about chronic kidney disease and ability of veterans to describe the tests used to detect chronic kidney disease. Validated instruments will be used [29,30], deployed via secure online surveys linked to the Kidney Score Platform's online interface to maximize data quality and security. We will also assess the impact of the Clinical Practice Toolkit on practitioners' perception of chronic kidney disease, chronic kidney disease knowledge, and important topics for chronic kidney disease patient education (Table 2).

Table 2. Patient and clinician behavior outcomes.

\begin{tabular}{ll}
\hline Outcome & Method of ascertainment \\
\hline Change in patient self-efficacy for disease self-management & Patient Activation Measure [28] \\
Change in patient perceived risk of chronic kidney disease and chronic kidney disease awareness & Boulware questionnaire [31] \\
Change in patient comfort communicating with providers & Stanford communication instrument [29] \\
Change in clinician perception of and actual chronic kidney disease knowledge & Knowledge survey \\
\hline
\end{tabular}

\section{Process Measures}

Using the Reach Evaluation-Adoption Implementation and Maintenance (RE-AIM) framework for program evaluation [32], we will conduct process evaluation of the Kidney Score's online platform and accompanying Clinical Practice Toolkit, across and within VA partnering sites. We will examine (1) reach to intended target audiences (veterans most at risk for chronic kidney disease, PACT team members); (2) effectiveness of the Kidney Score among veterans and practitioners, including usability of the online interface; (3) adoption of the Kidney Score and toolkit by primary care teams across and within partnering VA Medical Centers and (4) implementation consistency, costs, and adaptations made during deployment and delivery (Table 3). 
Table 3. Process outcomes for implementation analysis, based on the RE-AIM framework [32].

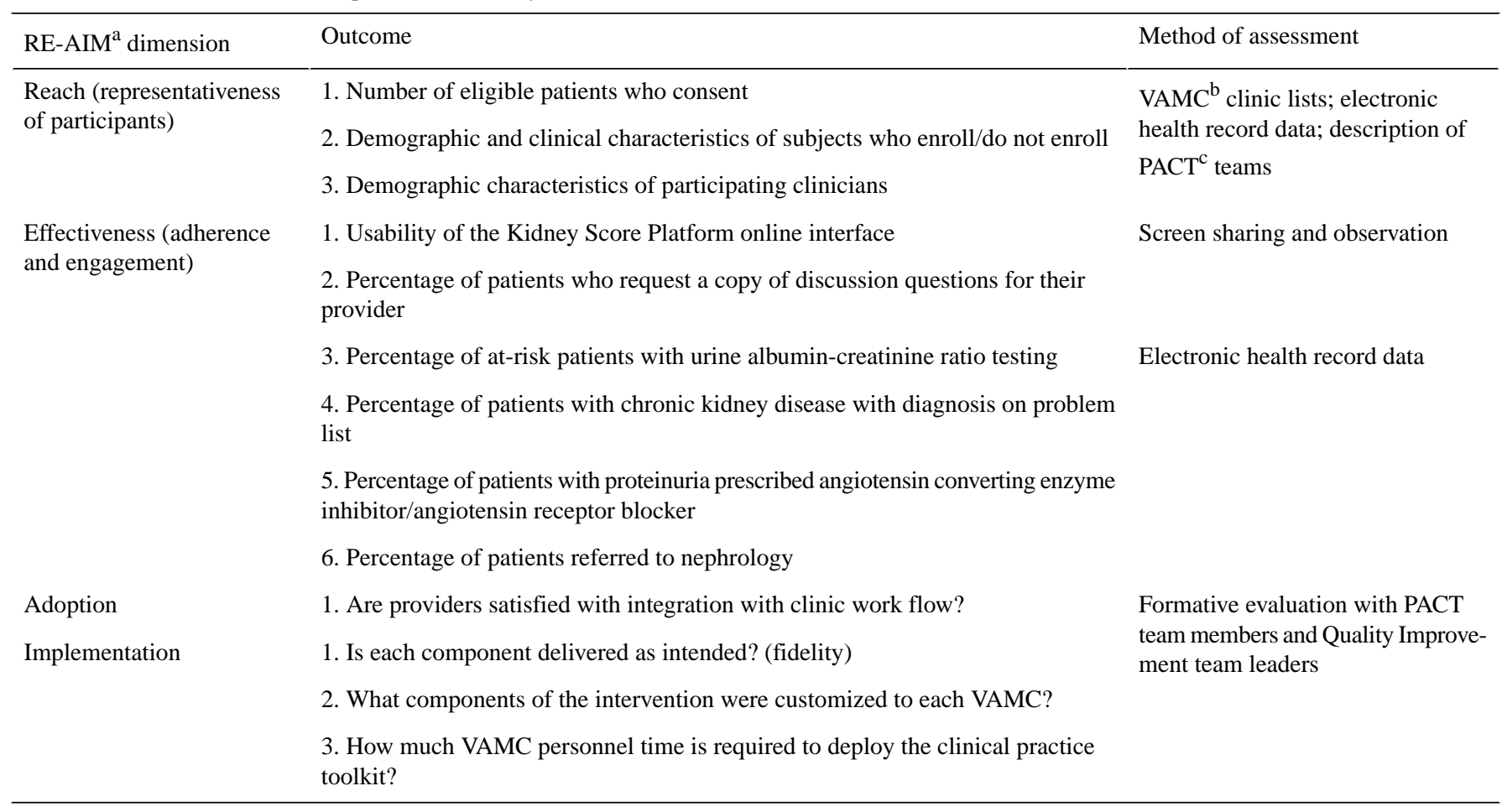

${ }^{a}$ RE-AIM: Reach Evaluation-Adoption Implementation and Maintenance.

${ }^{b}$ VAMC: Veteran Affairs Medical Center.

${ }^{\mathrm{c}}$ PACT: Patient Aligned Care Team.

\section{Sample Size Considerations}

The study is powered to detect changes in Patient Activation Measure (PAM) score, a validated measure of an individual's knowledge, skill, and confidence in managing one's health [28]. Data from prior self-management intervention studies have demonstrated a change in PAM score SD of 14.5. Using two-tailed paired $t$ test calculations, we would need to enroll 103 veterans to engage with the Kidney Score Platform and complete the pre and postsurveys to detect a 4-point clinically meaningful change in the PAM, assuming that the PAM would not change among individuals not exposed to Kidney Score Platform [33]. Accounting for a conservative 20\% dropout, we need to recruit 124 veterans. In previous studies [30] related to chronic kidney disease education in primary care, $67 \%$ of eligible patients agreed to participate. Preliminary data suggest that there are $>1200$ eligible veterans for our project in each medical center PACT program.

\section{Data Analysis}

Impact of the Kidney Score Platform on change in participant PAM scores (primary outcome) will be conducted with linear mixed models, adjusting for age, gender, diabetes status, kidney disease severity, and clinic site. Additional analyses will look for effect modification by clinician knowledge of chronic kidney disease. Similar methods will be used to assess secondary outcomes: self-reported communication with practitioners about chronic kidney disease and ability of veterans to describe the tests used to detect chronic kidney disease.

\section{Results}

As of September 2020, usability studies are underway with veterans and clinicians to refine the patient-facing components of the Kidney Score Platform before study initiation. Results and subsequent changes to the Kidney Score Platform will be published at a later date. The study is expected to be completed by December 2021 .

\section{Discussion}

This paper illustrates a theory-informed and evidence-based approach to developing and testing an intervention to enhance communication about kidney disease among patients and health care professionals. To develop all of the elements of the Kidney Score Platform, we leveraged the Behavior Change Wheel framework to directly target behaviors that were identified as barriers to optimal chronic kidney disease awareness and communication during preparatory interviews, using evidence-based intervention functions [19]. To test The Kidney Score Platform's impact, we partnered with VA leaders to identify the policy categories from the Behavior Change Wheel that could be employed at the organizational level to support its implementation. We are now poised to test the Kidney Score Platform's efficacy and effectiveness on increasing an individual's self-efficacy and activation to decrease chronic kidney disease risk and a primary care team's ability to manage chronic kidney disease and communicate about kidney disease.

While several chronic kidney disease education programs and awareness campaigns exist across the United States, there are 
limited examples in the literature of how theoretical frameworks can be leveraged to develop and test interventions to enhance kidney-related communication among clinicians and patients and improve self-efficacy and overall health among individuals with kidney disease. To our knowledge, this is one of the few chronic kidney disease awareness interventions developed and proposed to be tested according to an individual behavioral change theory (ie, Behavior Change Wheel). Theory-informed interventions are more likely to be effective than interventions not based on theory, though a formal study is required to test whether the slow deliberate process (such as that used to develop the Kidney Score Platform) leads to greater effectiveness within the context of health care delivery.

Results of the anticipated study examining the impact of Kidney Score Platform on patients and clinician chronic kidney disease awareness and communication will be shared with participants and will used to refine the different elements of the platform and their integration within primary care settings including new care delivery workflows, such as telehealth. Study results will be most applicable to care delivery in VA administration settings involving in-person and phone or video visits. While this is one limitation to this study, we anticipate that the Kidney Score Platform's impact on awareness, self-efficacy, educational, and communication outcomes among veterans and their clinicians will be generalizable to other settings and novel care delivery mechanisms. If so, the robust process by which the Kidney Score Platform was developed can serve as a model for the creation and implementation of other innovations that focus on behavior change as a means of enhancing kidney health. The refined Kidney Score Platform that will emerge from this study has the potential to significantly impact the lives of the approximately 37 million US adults affected by chronic kidney disease, as it will serve as a central component of the NKF's national public awareness initiative to educate, engage, and empower individuals at risk for and living with chronic kidney disease.

\section{Acknowledgments}

Lesley Hunter and Gary Bronz provided operational and administrative support.

\section{Conflicts of Interest}

None declared.

\section{Multimedia Appendix 1}

Kidney Score Platform design interview probe. [DOCX File, 24 KB-Multimedia Appendix 1]

\section{References}

1. Chronic kidney disease in the United States. Centers for Disease Control and Prevention. 2019. URL: https:/www.cdc.gov/ kidneydisease/publications-resources/2019-national-facts.html [accessed 2020-09-28]

2. 2018 USRDS annual data report: epidemiology of kidney disease in the United States. United States Renal Data System. Bethesda, MD: National Institutes of Health, National Institute of Diabetes and Digestive and Kidney Diseases; 2018. URL: https://www.usrds.org/annual-data-report/previous-adrs/ [accessed 2020-09-28]

3. Go A, Chertow G, Fan D, McCulloch C, Hsu C. Chronic kidney disease and the risks of death, cardiovascular events, and hospitalization. ACC Current Journal Review 2004 Dec;13(12):1296-1305. [doi: 10.1016/j.accreview.2004.11.016]

4. Sarnak MJ, Greene T, Wang X, Beck G, Kusek JW, Collins AJ, et al. The effect of a lower target blood pressure on the progression of kidney disease: long-term follow-up of the modification of diet in renal disease study. Ann Intern Med 2005 Mar 01;142(5):342-351. [doi: 10.7326/0003-4819-142-5-200503010-00009] [Medline: 15738453]

5. Inker LA, Astor BC, Fox CH, Isakova T, Lash JP, Peralta CA, et al. KDOQI US commentary on the 2012 KDIGO clinical practice guideline for the evaluation and management of CKD. Am J Kidney Dis 2014 May;63(5):713-735. [doi: 10.1053/j.ajkd.2014.01.416] [Medline: 24647050]

6. Vassalotti JA, Centor R, Turner BJ, Greer RC, Choi M, Sequist TD, National Kidney Foundation Kidney Disease Outcomes Quality Initiative. Practical approach to detection and management of chronic kidney disease for the primary care clinician. Am J Med 2016 Feb;129(2):153-162.e7 [FREE Full text] [doi: 10.1016/j.amjmed.2015.08.025] [Medline: 26391748]

7. Plantinga LC, Tuot DS, Powe NR. Awareness of chronic kidney disease among patients and providers. Adv Chronic Kidney Dis 2010 May;17(3):225-236 [FREE Full text] [doi: 10.1053/j.ackd.2010.03.002] [Medline: 20439091]

8. Greer RC, Cooper LA, Crews DC, Powe NR, Boulware LE. Quality of patient-physician discussions about CKD in primary care: a cross-sectional study. Am J Kidney Dis 2011 Apr;57(4):583-591 [FREE Full text] [doi: 10.1053/j.ajkd.2010.08.027] [Medline: 21131116]

9. Rothberg MB, Kehoe ED, Courtemanche AL, Kenosi T, Pekow PS, Brennan MJ, et al. Recognition and management of chronic kidney disease in an elderly ambulatory population. J Gen Intern Med 2008 Aug 29;23(8):1125-1130 [FREE Full text] [doi: 10.1007/s11606-008-0607-z] [Medline: 18443883]

10. Allen AS, Forman JP, Orav EJ, Bates DW, Denker BM, Sequist TD. Primary care management of chronic kidney disease. J Gen Intern Med 2011 Apr 5;26(4):386-392 [FREE Full text] [doi: 10.1007/s11606-010-1523-6] [Medline: 20922494] 
11. Szczech LA, Stewart RC, Su H, DeLoskey RJ, Astor BC, Fox CH, et al. Primary care detection of chronic kidney disease in adults with type-2 diabetes: the ADD-CKD Study (awareness, detection and drug therapy in type 2 diabetes and chronic kidney disease). PLoS One 2014 Nov 26;9(11):e110535 [FREE Full text] [doi: 10.1371/journal.pone.0110535] [Medline: 25427285]

12. Tuot DS, Plantinga LC, Hsu C, Jordan R, Burrows NR, Hedgeman E, et al. Chronic kidney disease awareness among individuals with clinical markers of kidney dysfunction. CJASN 2011 Jul 22;6(8):1838-1844. [doi: 10.2215/cjn.00730111]

13. Bodenheimer T, Lorig K, Holman H, Grumbach K. Patient self-management of chronic disease in primary care. JAMA 2002 Nov 20;288(19):2469-2475. [doi: 10.1001/jama.288.19.2469] [Medline: 12435261]

14. Plantinga LC, Boulware LE, Coresh J, Stevens LA, Miller ER, Saran R, et al. Patient awareness of chronic kidney disease: trends and predictors. Arch Intern Med 2008 Nov 10;168(20):2268-2275 [FREE Full text] [doi: 10.1001/archinte.168.20.2268] [Medline: 19001205$]$

15. Management of chronic kidney disease. VA/DoD Clinical Practice Guidelines. URL: https://www.healthquality.va.gov/ guidelines/CD/ckd/ [accessed 2020-09-01]

16. Chronic kidney disease surveillance system. Centers for Disease Control and Prevention. URL: $\underline{\text { http://www.cdc.gov/ckd }}$ [accessed 2020-09-01]

17. Azar A. Advancing American Kidney Health. U.S. Department of Health and Human Services. 2019. URL: https://www. asn-online.org/policy/webdocs/AdvancingAmericanKidneyHealth-1.pdf [accessed 2020-09-28]

18. Glasgow R, Chambers D. Developing robust, sustainable, implementation systems using rigorous, rapid and relevant science. Clin Transl Sci 2012 Feb;5(1):48-55 [FREE Full text] [doi: 10.1111/j.1752-8062.2011.00383.x] [Medline: 22376257]

19. Michie S, van Stralen MM, West R. The behaviour change wheel: a new method for characterising and designing behaviour change interventions. Implement Sci 2011 Apr 23;6(1):42 [FREE Full text] [doi: 10.1186/1748-5908-6-42] [Medline: 21513547]

20. Diamantidis CJ, Becker S. Health information technology (IT) to improve the care of patients with chronic kidney disease (CKD). BMC Nephrol 2014 Jan 09;15(1):7 [FREE Full text] [doi: 10.1186/1471-2369-15-7] [Medline: 24405907]

21. Baranowski T, Buday R, Thompson DI, Baranowski J. Playing for real: video games and stories for health-related behavior change. Am J Prev Med 2008 Jan;34(1):74-82 [FREE Full text] [doi: 10.1016/j.amepre.2007.09.027] [Medline: 18083454]

22. Stout P, Villegas J, Kim H. Enhancing learning through use of interactive tools on health-related websites. Health Educ Res 2001 Dec;16(6):721-733. [doi: 10.1093/her/16.6.721] [Medline: 11780710]

23. Are you the 33\%? National Kidney Foundation. URL: https://www.kidney.org [accessed 2020-09-01]

24. CKDinform 2.0: chronic kidney disease in primary care: implementation best practices to improve outcomes. National Kidney Foundation. URL: https://education.kidney.org/ckdi2enduring [accessed 2020-09-01]

25. Agency for Health care Research and Quality. Health Literacy Measurement Tools (Revised). URL: http://www.ahrq.gov/ professionals/quality-patient-safety/quality-resources/tools/literacy/index.html [accessed 2020-09-01]

26. Chronic kidney disease change package. National Kidney Foundation. URL: https://www.kidney.org/contents/ chronic-kidney-disease-change-package [accessed 2020-09-01]

27. Hypertension control change package for clinicians. Centers for Disease Control and Prevention. 2015. URL: https:/ /millionhearts.hhs.gov/files/HTN_Change_Package.pdf [accessed 2020-09-28]

28. Hibbard JH, Mahoney ER, Stockard J, Tusler M. Development and testing of a short form of the patient activation measure. Health Serv Res 2005 Dec;40(6 Pt 1):1918-1930 [FREE Full text] [doi: 10.1111/j.1475-6773.2005.00438.x] [Medline: 16336556]

29. Lorig K, Stewart A, Ritter P, Gonzalez V, Laurant D, Lynch J. Outcome Measures for Health Education and Other Health Care Interventions. Thousand Oaks, CA: Sage Publications; 1996.

30. Tuot DS, Zhu Y, Velasquez A, Espinoza J, Mendez CD, Banerjee T, et al. Variation in patients' awareness of CKD according to how they are asked. Clin J Am Soc Nephrol 2016 Sep 07;11(9):1566-1573. [doi: 10.2215/CJN.00490116] [Medline: 27340288]

31. Boulware LE, Carson KA, Troll MU, Powe NR, Cooper LA. Perceived susceptibility to chronic kidney disease among high-risk patients seen in primary care practices. J Gen Intern Med 2009 Oct 27;24(10):1123-1129 [FREE Full text] [doi: 10.1007/s11606-009-1086-6] [Medline: 19711135]

32. Glasgow RE, Klesges LM, Dzewaltowski DA, Estabrooks PA, Vogt TM. Evaluating the impact of health promotion programs: using the RE-AIM framework to form summary measures for decision making involving complex issues. Health Educ Res 2006 Oct 15;21(5):688-694. [doi: 10.1093/her/cyl081] [Medline: 16945984]

33. Hibbard JH, Greene J, Tusler M. Improving the outcomes of disease management by tailoring care to the patient's level of activation. Am J Manag Care 2009 Jun;15(6):353-360 [FREE Full text] [Medline: 19514801]

\section{Abbreviations}

COM-B: capability, opportunity, motiviation - behavior

NKF: National Kidney Foundation

PACT: Patient Aligned Care Teams 
PAM: Patient Activation Measure

RE-AIM: Reach Evaluation-Adoption Implementation and Maintenance

VA: Veterans Affairs

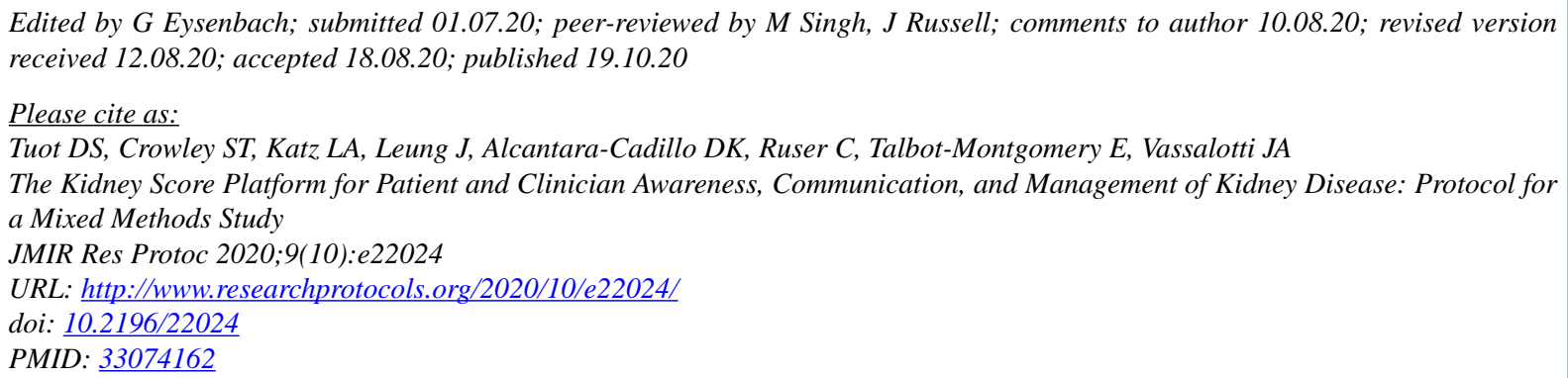

CDelphine S Tuot, Susan T Crowley, Lois A Katz, Joseph Leung, Delly K Alcantara-Cadillo, Christopher Ruser, Elizabeth Talbot-Montgomery, Joseph A Vassalotti. Originally published in JMIR Research Protocols (http://www.researchprotocols.org), 19.10.2020. This is an open-access article distributed under the terms of the Creative Commons Attribution License (https://creativecommons.org/licenses/by/4.0/), which permits unrestricted use, distribution, and reproduction in any medium, provided the original work, first published in JMIR Research Protocols, is properly cited. The complete bibliographic information, a link to the original publication on http://www.researchprotocols.org, as well as this copyright and license information must be included. 